\title{
Coexistence of Dural and Intraventricular Meningioma-A Rare Entity
}

\author{
Rajul Rastogi*, Yuktika Gupta, Satish Pathak, Vijai Pratap and Neha \\ Department of Radiodiagnosis, Teerthanker Mahaveer Medical College \& Research Center, India
}

*Corresponding author: Rajul Rastogi, Department of Radiodiagnosis, Teerthanker Mahaveer Medical College \& Research Center, Moradabad, Uttar Pradesh, India

\begin{abstract}
Meningioma is a commonly encountered solitary, benign, intracranial, extra-axial tumor in day-to-day brain imaging. Majority are dural-based located commonly at the cerebral convexities. Uncommonly, they are multiple or may be seen in intraventricular location. Coexisting dural and intraventricular meningioma is an exceedingly rare entity. It may be difficult to differentiate them from metastases. Hence through this case report, we attempt to describe this rare entity with its differentiation from similarappearing lesions.
\end{abstract}

Keywords: Coexistence; Dural; Intraventricular; Meningioma

\section{Introduction}

Meningiomas are extra-axial tumors that are majorly benign and constitute nearly one-quarter of all intracranial tumors [1]. They are categorized in to Grade I (benign), Grade II (atypical) and Grade III (anaplastic) according to World Health Organisation Classification 2016 [2]. Atypical meningiomas constitute up to one-third of all meningiomas [3]. Multifocal meningiomas are uncommon occurring in less than a tenth of meningioma cases [4]. Meningioma occurring within the ventricular system are very rare comprising of only $0.5-3.7 \%$ cases of meningioma epic entered primarily in the lateral ventricles especially the trigone arising from the tela choroidae and choroid plexus stroma $[5,6]$. Very few case reports of atypical and anaplastic intraventricular meningioma exist in medical literature [5-9]. Hence in this case report, we describe a rare coexistence of dural and intraventricular, atypical meningiomas preoperatively diagnosed on imaging.

\section{Case Report}

A 65year old man with history of chronic headache since more than nine months came for a magnetic resonance imaging (MRI) of the brain to exclude organic lesion. Routine laboratory tests including those in blood and urine were unremarkable. MRI brain revealed two similar-appearing extra-axial lesions, one arising from the dura mater along the lateral convexity of left frontal lobe with broad dural base measuring up to $45^{*} 43 * 39 \mathrm{~mm}$ and another in the occipital horn of the left lateral ventricle measuring to $46 * 41 * 40$ $\mathrm{mm}$. The lesions were irregular in shape with lobulated margins and heterogeneous internal architecture. There was significant edema around both the lesions along with significant mass effect causing partial effacement \& contralateral subfalcine herniation of left lateral ventricle, dilatation of right lateral ventricle and midline shift of approximately 8-9 $\mathrm{mm}$ to right side.

Both lesions appeared nearly isointense to gray matter with irregular areas of hyperintensity within on T1-weighted images (Figure 1). T2-weighted images revealed internal heterogeneity of lesions, large internal hyperintense areas and perilesional edema more conspicuously with multifocal areas of indistinct tumor-brain interface around the circumference (Figure 2). Solid nature of the two lesions along with internal heterogeneity and perilesional edema was also well demonstrated on T2LFLAIR images (Figure $3)$. Diffusion weighted images along with ADC maps revealed heterogeneous areas of restricted diffusion confirming high cellularity of the lesions (Figure 4). Irregular areas of calcification were noted in both tumorallesions on susceptibility weighted images (Figure 5). Postcontrast T1GRE images revealed heterogeneous postcontrast enhancement with signs of internal necrosis along with multiple dilated vascular channels in perilesional location \& 
enhancing dural tail associated with dural meningioma but brain invasion with both lesions (Figure 6). MR spectroscopy revealed high levels of alanine with nearly no identifiable NAA (Figure 7). Based on the above findings, the diagnosis of coexisting dural frontal and left ventricular atypical meningiomas was made which was confirmed on histopathology following surgical excision of both tumors. The Ki-67 index was approximately 18-22\%. The patient was referred to radiotherapy and chemotherapy at a higher center for further management.

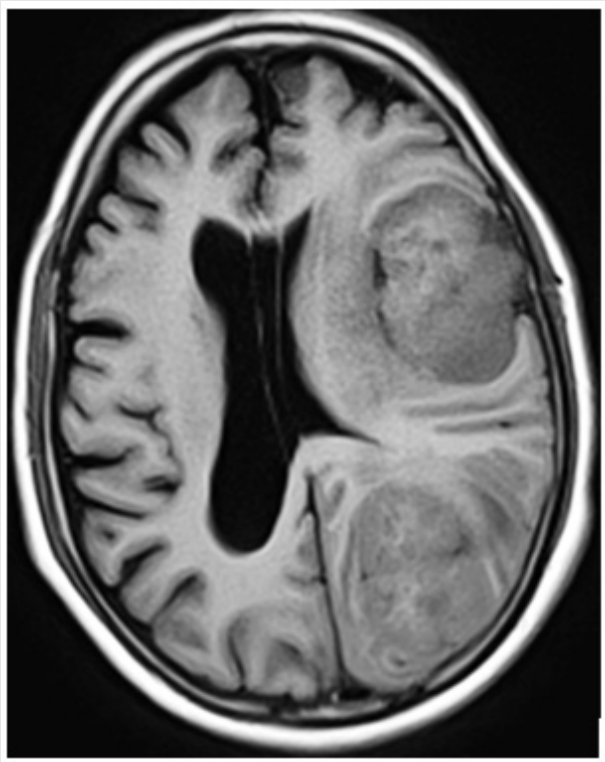

Figure 1: Axial T1W MR image shows coexisting dural and intraventricular meningiomas on left side of midline with internal hyper densities.

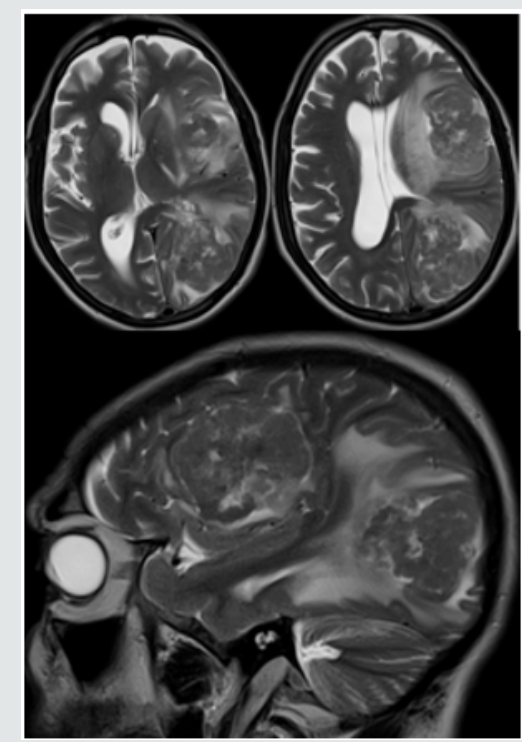

Figure 2: Axial (upper row) and sagittal (lower row) T2W MR images show internal heterogeneity, perilesional edema and signs of brain invasion in coexisting dural and intraventricular meningiomas on left side of midline.

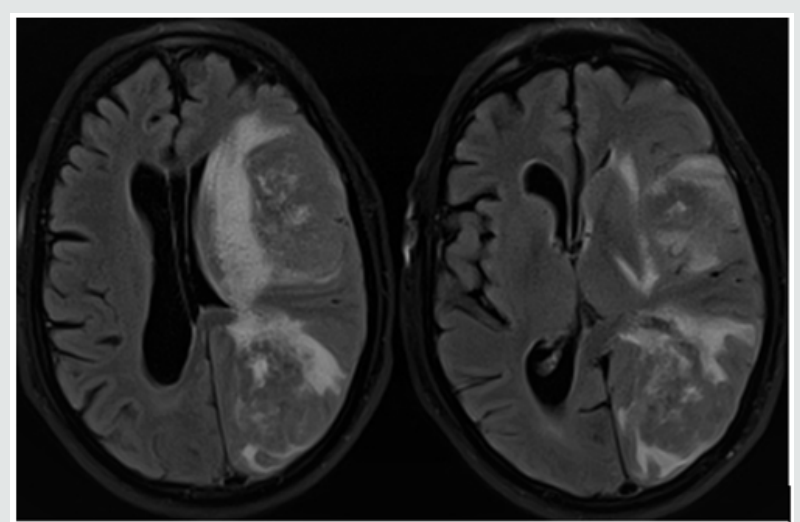

Figure 3: Axial T2FLAIR MR image show the solid nature, internal heterogeneity \& significant perilesional edema with coexisting dural meningioma and intraventricular meningiomas on left side of midline.

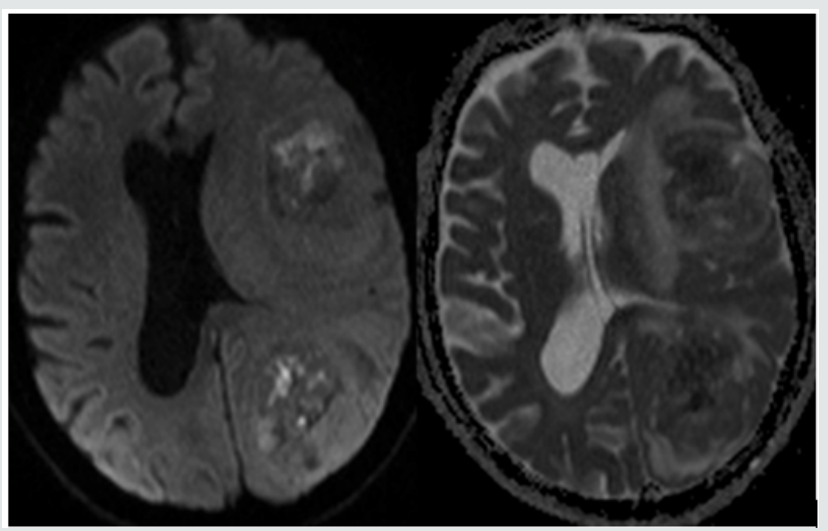

Figure 4: Axial DW (Left) MR image with ADC map (Right) show heterogeneous areas of restricted diffusion within coexisting dural meningioma and intraventricular meningiomas on left side of midline.

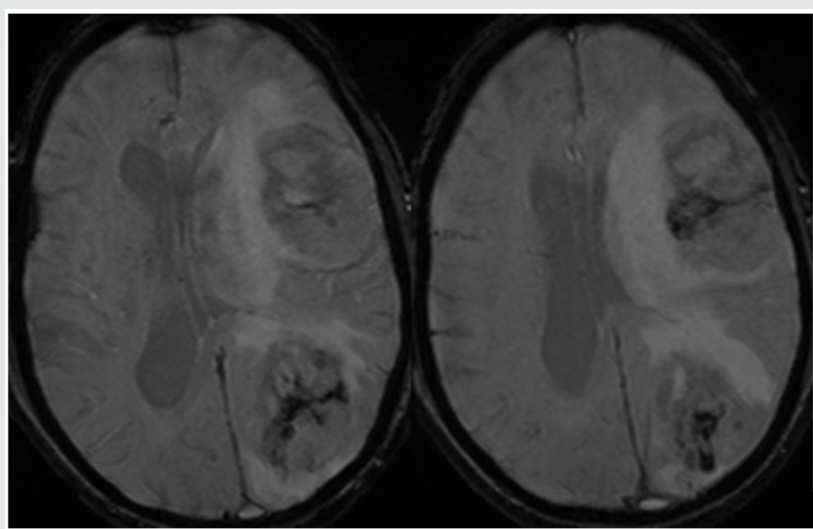

Figure 5: Susceptibility-Weighted MR images showing heterogeneous areas of calcification within coexisting dural meningioma and intraventricular meningiomas on left side of midline. 


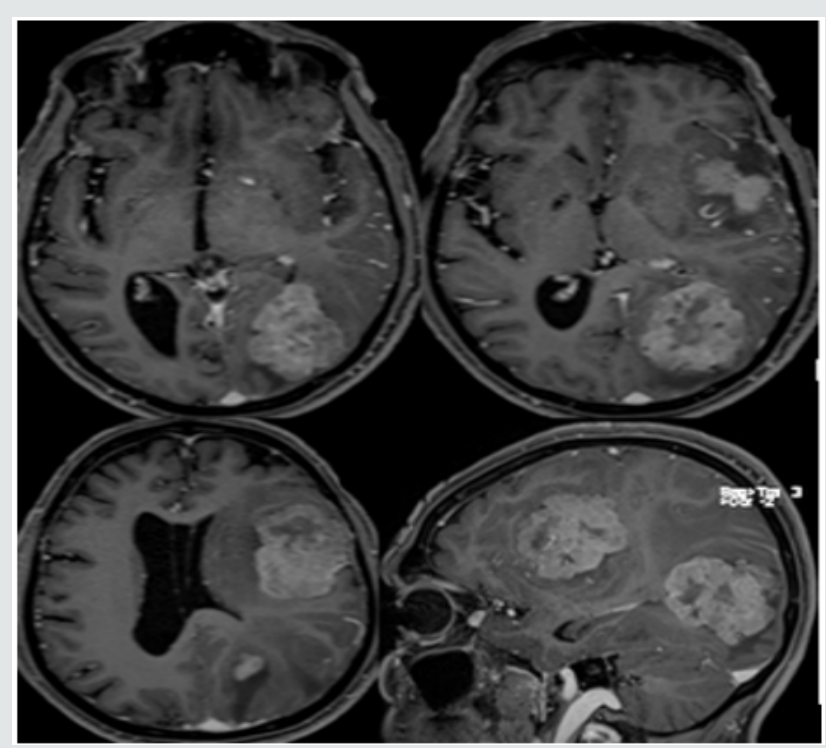

Figure 6: Postcontrast axial \& sagittal (lower right) T1GRE MR images show heterogeneous enhancement, multiple vascular channels, areas of necrosis, dural and brain invasion in coexisting dural meningioma and intraventricular meningiomas on left side of midline.

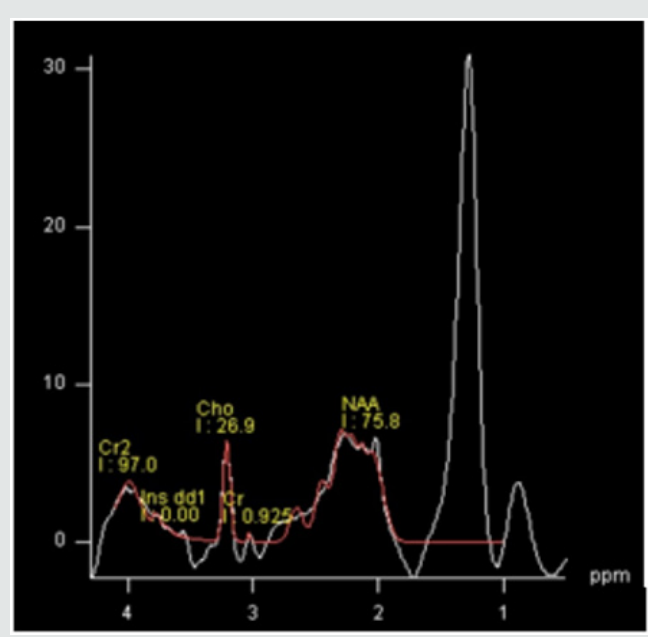

Figure 7: MR spectra at low TE (35msec) shows a high alanine peak at 1.3-1.4ppm within meningioma.

\section{Discussion}

The incidence of meningioma is apparently rising not only due to improved and extensive neuroimaging but also due to increased proportion of the elderly population where it has the common occurrence [10]. The higher incidence of meningioma in adult females reported in recent literature in contrast to previous reports of male preponderance is highly attributed to various reproductive and hormonal factors [11]. Arachnoid cap cells are the usual sites for origin of most meningiomas with majority being benign. Less percentage of meningioma show signs of malignancy like marked vascularity; lack of internal organoid architecture; higher percentage of mitotic figures, nuclear pleomorphism; disproportionately large nucleolus, focal necrosis and infiltration in to brain parenchyma along with variable expression of Ki-
67, a marker for tumor recurrence in atypical and anaplastic meningiomas $[10,12,13]$.

There are increasing reports of benign, multiple meningiomas in recent literature which account for nearly one-tenth of all cases $[4,14]$. Tumor cell spread by CSF or hematogenous dissemination is the most widely accepted mechanism making two different sporadic forms in one patient very rare [10]. Previous reports have revealed atypical with psammomatous and fibrous with anaplastic meningiomas $[10,15,16]$. But ours is probably the first report of double atypical meningioma, one dural and another intraventricular in location. Also, very few reports of atypical intraventricular meningioma exist in the literature [17]. Atypical meningioma shows progression to anaplastic meningioma within a short period of time [17]. Very rarely meningioma may occur in 
intraparenchymal location within the cortex where the absence of dural tail may make it difficult to distinguish it from high-grade glioma, cavernoma angioma, metastases and sarcoma [18].

Multiple meningioma may rarely present as bipolar disorder like psychiatric illness making imaging mandatory to rule out organic lesion prior to targeted medical management of bipolar psychotic illness [19]. Multiple dural-based meningioma also forms a differential diagnosis of multiple dural-based metastases [20]. Classical benign meningioma appears as a homogeneous, round to ovoid, dural-based, extra-axial lesion with sharp margins, internal calcification and near-homogeneous postcontrast enhancement with or without bone reaction. Atypical radiological features include intra-tumoral heterogeneity, presence of internal hemorrhage or necrosis, venous sinus invasion, lack of tumor capsule enhancement, indistinct tumor-brain interface, diffusion restriction, increased Ktrans on dynamic contrast MR examination and peritumoral edema with delayed increased vascularity causing significant mass effect $[21,22]$. High and low-intensity large areas corresponding to internal hemorrhage and necrosis respectively along with non-spheroid shape, significant calcification and bone destruction are signs of high-grade meningioma $[21,22]$.

Distinguishing the grade of meningioma is critical for management. Surgical excision of tumor along with invaded dura is the standard mode of treatment for WHO Grade I meningioma while supplementary radiotherapy and chemotherapy is needed for Grade II \& III meningiomas [10].

\section{Conclusion}

Meningioma is a common benign and solitary intracranial tumor presenting in elderly females. Uncommonly, it may atypical or anaplastic showing signs of malignancy. Multiple meningiomas at dural \& intraventricular locations are exceedingly rare. Radiological imaging plays an important role not only in detecting coexisting multiple meningioma but also in diagnosing its correct grade, a prerequisite for optimal management.

\section{References}

1. Louis DN, Scheithauer BW, Budka H, Von Deimling A, Kepes JJ (2000) Meningiomas. Pathology and Genetics of tumors of the nervous system: World Health Organisation Classification of Tumors. In: Kleihues P, Cavenee WK (Eds.), IARC Press, Lyon, PP: 176-184.

2. Louis DN, Perry A, Reifenberger G, Von Deimling A, Figarella Branger D, et al. (2016) The 2016 World Health Organization Classification of Tumors of the Central Nervous System: a summary. Acta Neuropathol 131(6): 803-820

3. Rogers L, Gilbert M, Vogelbaum MA (2010) Intracranial meningioma of atypical (Grade II) histology. J Neurooncol 99(3): 393-405.

4. Tsermoulas G, Turel MK, Wilcox JT, Shultz D, Farb R, et al. (2018) Management of multiple meningiomas. J Neurosurg 128(5): 1403-1409.
5. Kim EY, Kim ST, Kim HJ, Jeon P, Kim KH, et al. (2009) Intraventricular meningiomas: radiological findings and clinical features in 12 patients. Clin Imaging 33(3): 175-180.

6. Menon G, Nair S, Sudhir J, Rao R, Easwer HV, et al. (2009) Meningiomas of the lateral ventricle-a report of 15 cases. Br J Neurosurg 23(3): 297 303.

7. Cleary C, Curtin D (2010) Giant atypical intraventricular meningioma presenting with visual loss in a child. Ir J Med Sci 179: 617-619.

8. Garcia Conde M, Roldan Delgado H, Martel Barth Hansen D, Manzano Sanz C (2009) Anaplastic transformation of an atypical intraventricular meningioma with metastases to the liver: case report. Neurocirugia (Astur) 20(6): 541-549.

9. Krayenbühl N, Pravdenkova S, Al Mefty O (2007) De novo versus transformed atypical and anaplastic meningiomas: comparisons of clinical course, cytogenetics, cytokinetics, and outcome. Neurosurgery 61(3): 495-503.

10. Liu Y, Song DP, Wang T (2017) Meningiomas with different histological grade in the same patient. Medicine (Baltimore) 96: 50(e9086).

11. Splavski B, Hadzic E, Bagic I, Vrtaric V, Splavski B (2017) Simple tumor localisation scale for estimating management outcome in intraventricular meningioma. World Neurosurg 104: 876-882.

12. Ozkok pala EE, Kucuk U, Bayol U, Keskin E, Cakir E, et al. (2014) Histopathological review of meningiomas: 125 cases. Journal of Neurological Sciences 31(4): 699-708.

13. Morishita A, Kondo T, Ehara K, Tamaki N, Kodama Y (2002) Papillary meningioma demonstrate a progressive increase in MIB-1 index: a case report. Japanese Journal of Neurosurgery 11(8): 530-535.

14. Ojo A, Fynn E (2006) Multiple meningiomas: case report. South African J Radiol 10(2): 21-23.

15. Koh YC, Yoo H, Whang GC, Kwon OK, Park HI (2001) Multiple meningiomas of different pathological features: case report. J Clin Neurosci 8(Suppl1): 40-43.

16. Tomita T, Kurimoto M, Yamatani K, Nagai S, Kuwayama N, et al. (2003) Multiple meningiomas consisting of fibrous meningioma and anaplastic meningioma. J Clin Neurosci 10(5): 622-624.

17. Kim HD, Choi CY, Lee DJ, Lee CH (2011) Intraventricular Atypical Meningiomas. J Korean Neurosurg Soc 49: 292-295.

18. Jadik S, Stan AC, Dietrich U, Peitila TA, Elsharkawy AE (2014) Intraparenchymal meningioma mimicking cavernous malformation: a case report and review of the literature. Journal of Medical Case Reports 8: PP 467.

19. Mahapatra A, Sood M, Khandelwal SK (2016) Multiple Meningioma in a Patient of Bipolar Disorder: The Dilemma of Detecting Structural Brain Lesions in the Backdrop of a Long-Standing Psychiatric Illness. Journal of Clinical and Diagnostic Research 10(8): VD01-VD02.

20. Manganaro MS, Millet JD, Maganaro MS (2019) Multifocal meningioma mimicking dural-based leptomeningeal metastases at 18F-Fluciclovine PET/CT. Clin Nucl Med 44(7): 594-595.

21. Coroller TP, Bi WL, Huynh E, Abedalthagafi M, Aizer AA, et al. (2017) Radiographic prediction of meningioma grade by semantic and radiomic features. PLoS One 12(11): e0187908.

22. Kunimatsu A, Kunimatsu N, Kamiya K, Katsura M, Mori H, et al. (2016) Variants of meningiomas: a review of imaging findings and clinical features. Jpn J Radiol 34(7): 459-469. 
(C) (P) This work is licensed under Creative Commons Attribution 4.0 License

To Submit Your Article Click Here: Submit Article

DOI: $10.32474 /$ JJNBD.2020.04.000180

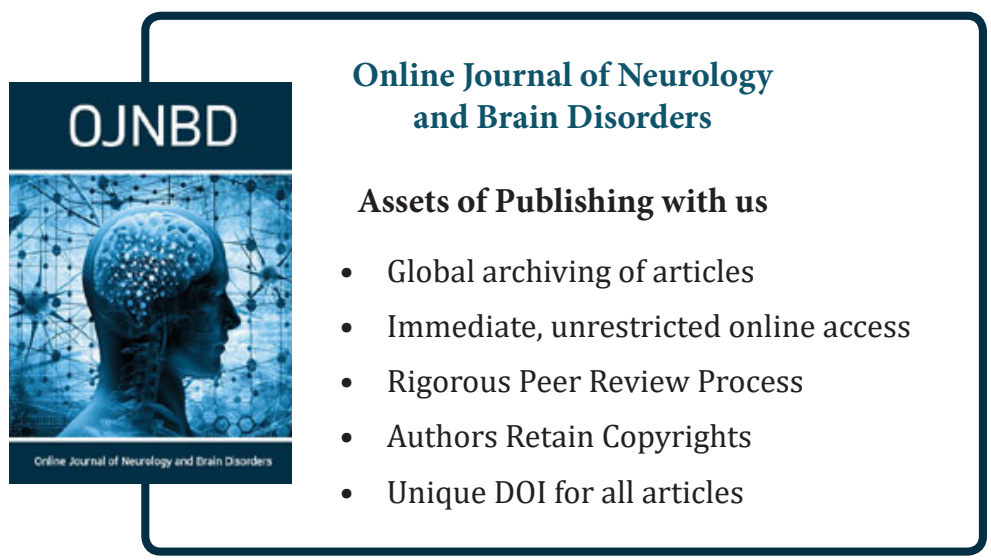

